\title{
Power and Delay Optimal Transmission Scheduling: Small Delay Asymptotics
}

\author{
Randall Berry \\ Dept. of ECE, Northwestern University \\ 2150 Sheridan Rd. \\ Evanston, IL 60201, USA \\ e-mail: rberry@ece.northwestern.edu
}

\section{SUMmARY}

For wireless data traffic, energy efficiency can be improved by adapting the transmission power and rate over time based on the offered traffic as well as any channel state information. In this context, a variety of transmission scheduling approaches have been studied including $[1,2,3]$. These approaches attempt to manage the fundamental trade-off between packet delay and transmission power or energy. Specifically, packet delay can be reduced by transmitting at a higher rate, but this requires an increased energy per bit. In fading channels, reducing packet delay also prohibits users from optimally allocating their power over time in response to channel variations.

In this paper, we consider a model for transmission scheduling over a fading channel from [2]. In this model, data randomly arrives at a transmission buffer where it is held until it is encoded and transmitted over the fading channel. The transmitter can vary the transmission power and rate based on both the channel state and the buffer occupancy. In [2], the optimal trade-off between the average delay incurred by the arriving data and the long-term average power was studied in a Markov decision framework. The behavior of this trade-off was characterized in the asymptotic regime of large delays (low power). In this regime, it was shown that the rate at which the required power decreases as the average delay, $D$, increases is ${ }^{1} \Theta\left(\frac{1}{D^{2}}\right)$.

In this paper, we focus on the behavior of the power/delay trade-off in the asymptotic regime of small delays (high power). We focus on a case where infinite power is required to minimize the average delay. This will be true for example in a Rayleigh fading channel. In this regime, we show that compared to the large delay asymptotics, the average power decreases at a much faster rate as the delay increases. This implies that the savings in power gained by relaxing the delay constraint are much more significant when the delay constraint is stringent.

We consider a discrete-time fluid queueing model with an infinite buffer size. At time $n, U_{n}$ bits are removed from the buffer and transmitted over a block fading channel, with channel gain $H_{n}$, assumed to be known at the transmitter. The transmission power required to reliably send these bits is given by $P\left(H_{n}, U_{n}\right)=\frac{\sigma^{2}}{\left|H_{n}\right|^{2}}\left(2^{U_{n} / N}-1\right)$, where $N$ is the number of channel uses in each block and $\sigma^{2}$ is the additive noise variance. Let $\mu(s, h)$ denote a transmission policy, that specifies the amount of data transmitted at each time-unit as a function of the current buffer state, $S_{n}$ and the current channel

\footnotetext{
${ }^{1}$ We use the notation $a_{n}=O\left(b_{n}\right)$ if $\lim _{\sup } \rightarrow \infty \frac{\left|a_{n}\right|}{\left|b_{n}\right|}<\infty$, $a_{n}=\Omega\left(b_{n}\right)$ if $\lim \sup _{n \rightarrow \infty} \frac{\left|b_{n}\right|}{\left|a_{n}\right|}<\infty$ and $a_{n}=\Theta\left(b_{n}\right)$ if $a_{n}=O\left(b_{n}\right)$ and $a_{n}=\Omega\left(b_{n}\right)$.
}

gain $H_{n}$. For a given policy $\mu$, let $\bar{D}^{\mu}$ denote the average delay and let $\bar{P}^{\mu}$ denote the long-term average transmission power. For a given channel and arrival process, the optimal delay/power curve, $D^{*}(P)$ is defined to be

$$
D^{*}(P)=\inf \left\{\bar{D}^{\mu}: \mu \text { such that } \bar{P}^{\mu} \leq P\right\} .
$$

For the channels of interest as $P \rightarrow \infty, D^{*}(P) \rightarrow D_{\text {min }}$ and for any $P<\infty, D^{*}(P)>D_{\min }$. We study the rate at which $D^{*}(P)$ decreases.

First we consider a simple "fixed-power" transmission policy in which the transmitter always sends at a constant power $\bar{P}$, but a variable rate.

Proposition 1 For any sequence of fixed power policies, $\mu_{k}$, with $\bar{P}^{\mu_{k}} \rightarrow \infty, \bar{D}^{\mu_{k}}-D_{\text {min }}=O\left(\frac{1}{\log P^{\mu_{k}}}\right)$. Furthermore, assuming that the channel gain distribution satisfies $\operatorname{Pr}\left(|H|^{2}<\right.$ $h)=\Theta(h)$ as $h \rightarrow 0$, then $\bar{D}^{\mu_{k}}-D_{\min }=\Omega\left(\frac{1}{\bar{P}^{\mu_{k}} \log \bar{P}^{\mu_{k}}}\right)$.

The proof of this relies on finding upper and lower bounds on the average buffer occupancy and then applying Little's law. The bounds used are similar to Kingmann's bounds for a $\mathrm{G} / \mathrm{G} / 1$ queue [4].

Next we consider channel threshold policies, where the transmitter sends at a constant rate $R$ whenever the magnitude of the channel gain is greater than a given threshold and transmits nothings otherwise.

Proposition 2 There exists a sequence of channel threshold policies, $\left\{\mu_{k}\right\}$ with $\bar{P}^{\mu_{k}} \rightarrow \infty$ and $D^{\mu_{k}}-D_{\min }=\Theta\left(e^{-\bar{P}^{\mu_{k}}}\right)$.

From this it follows that $D^{*}(P)$ must decrease faster than $e^{-P}$ as $P \rightarrow \infty$.

These propositions can be generalized to a multi-user setting as well as a finite buffer model.

\section{ACKnowledgments}

The author wishes to thank Vijay Subramanian for several useful discussions and the reviewers for their helpful comments.

\section{REFERENCES}

[1] B. Collins and R. Cruz, "Transmission Policies for Time Varying Channels with Average Delay Constraints," in Proc. 1999 Allerton Conf. on Commun. Control, \& Comp., (Monticello, IL), 1999.

[2] R. Berry and R. Gallager, "Communication over fading channels with delay constraints," IEEE Trans. on Information Theory, vol. 48, pp. 1135-1149, May 2002.

[3] B. Prabhakar, E. Uysal-Biyikoglu, and A. E. Gamal, "Energyefficient transmission over a wireless link via lazy packet scheduling," in Proc. IEEE Infocom 2001, 2001.

[4] L. Kleinrock, Queueing Systems, vol. II. John Wiley and Sons, 1976. 